\title{
Editorial
}

\section{LOW X 2013}

\author{
Christophe Royon ${ }^{1, *}$, Alexander Milov ${ }^{2}$ and Michael Lublinsky ${ }^{3}$
}

\author{
${ }^{1}$ DSM-IRFU-SPP, CEA Saclay, France; ${ }^{2}$ Weitzman Institute of Science, Rehovot, Israel; ${ }^{3}$ Ben Gurion University, Beer \\ Sheva, Israel
}

The "low x 2013" workshop organized in collaboration between the Weitzman Institute of Science (Rehovot), the Neguev University and CEA Saclay in France (Direction des Sciences de la Matiere, Institut de Recherche Fondamentale de l'Univers and Institut de Physique Theorique) took place between May 30 and June 4 in the Weitzman Institute of Science, Rehovot and Eilat. The spirit of this meeting was to favour fruitful and informal discussions and new collaborations between theorists and experimentalists in the domain of Particle Physics. Physicists coming from all over the world presented and discussed the most recent experimental and theoretical results related to Quantum Chromodynamics the theory which describes the structure of the proton in terms of quark and gluons.

The timing of the workshop was ideal after the recent start of the Large Hadron Collider at CERN which allows us to accelerate protons at almost the speed of light and reach a new unprecedented collision energy close to the big bang conditions. New results are presently obtained by the different collaborations at CERN, Geneva. Special domains are of interest for this workshop. For instance, "low x" physics can lead to the observation of new states of matter at the LHC, such as the quark-gluon plasma or the so called Colour Glass condensate. Other domains such as a better understanding of the proton structure in terms of gluons and quarks as well as the study and measurement of very special events where high-emergy protons are found to be intact after the interaction are of special interest.

Almost 100 physicists from all over the world came to the meeting and ample of time was dedicated to discussion sessions where ideas can be exchanged and new experimental methods and theoretical ideas can be pursued. Typically, there was a 2-hour discussion every day. Thanks to the generous support of the Science Division of the French Embassy in Tel Aviv, we were able to invite many participants of France which allowed us to reinforce the cooperation between Israel and France in the domain of fundamental research, which was abolutely necessary in high energy physics where large accelerators as the LHC requires such a collaboration.

The workshop went on in a friendly atmosphere - even some "hot" discussions happened between physicists who did not agree to the interpretation of some results, but this is the way science makes progress. Last but not least, common discussions and interactions between physicists were pursued during lunches, dinners and the tour on Saturday in Jerusalem and the Dead Sea that drove us between Rehovot and Eilat which were also the fundamental aspects of the workhop in order to establish long term contacts and collaborations between physicists.

C Royon; Licensee Bentham Open.

This is an open access article licensed under the terms of the Creative Commons Attribution Non-Commercial License (http://creativecommons.org/licenses/ by-nc/3.0/) which permits unrestricted, non-commercial use, distribution and reproduction in any medium, provided the work is properly cited. 